\section{Defect Transition Can Enhance Band-Edge Emission in Semiconductor Nanocomposites}

Defects are usually considered a big disadvantage in solid-state emitters because they reduce the quantum efficiency of bandgap emission. However, researchers at National Taiwan University have found that coating a layer of CdSe/ZnS core-shell nanoparticles can convert the defect loss into bandgap emission in $\mathrm{ZnO}$ nanorods. The bandgap emission of $\mathrm{ZnO}$ nanorods can be enhanced as much as 30 times. The possible mechanism is based on the resonance effect between defect transition and band-to-band excitation and the transfer of excited electrons between conduction band edges.

In the March 15, 2008, issue of Optics Letters (DOI: 10.1364/OL.33.000569 p. 569), L.-J. Tzeng, C.-L. Cheng and Y.-F. Chen reported preparing $\mathrm{ZnO}$ nanorods on the surface of gold-coated sapphire using a mixture of high-purity $\mathrm{ZnO}$ and carbon powders by a vaporliquid-solid process. The nanorods were well aligned and had an average diameter of $50 \mathrm{~nm}$ and a length of about $1 \mu \mathrm{m}$. The researchers used a sol-gel process to synthesize CdSe/ZnS core-shell quantum dots. First, CdSe cores were made using $\mathrm{CdO}$ and Se precursors in stearic acid at $320^{\circ} \mathrm{C}$ in the presence of trioctylphosphine oxide and hexadecylamine. Then, the researchers used diethylzinc and hexamethyldisilathiane as the $\mathrm{Zn}$ and $\mathrm{S}$ precursors to form a $\mathrm{ZnS}$ shell on the CdSe cores. Finally, the core-shell quantum dots dissolved in methylbenzene were spin-coated onto the $\mathrm{ZnO}$ nanorods.

After the preparation, the researchers used a spectrometer equipped with a $\mathrm{He}-\mathrm{Cd}(325 \mathrm{~nm})$ laser source, a Spex $0.85 \mathrm{~m}$ double-grating monochromator, and a cooled photomultiplier tube to obtain the photoluminescence spectra of pure $\mathrm{ZnO}$ nanorods and CdSe/ZnS quantum dots, and the composite of $\mathrm{ZnO}$ nanorods coated with CdSe/ZnS quantum dots. They found that the weak bandgap emission of $\mathrm{ZnO}$ nanorods at $380 \mathrm{~nm}$ is enhanced in the composite, while the emission of $\mathrm{CdSe} / \mathrm{ZnS}$ quantum dots at $570 \mathrm{~nm}$ is reduced. This indicates that energy is transferred from CdSe/ZnS quantum dots to the $\mathrm{ZnO}$ nanorods where it is converted into the bandgap emission. The researchers concluded that the defect emission at $540 \mathrm{~nm}$ should be in resonance with the bandgap excitation of the quantum dots to obtain the most efficient energy conversion. When they prepared samples coated with core-shell CdSe/
ZnS quantum dots prepared such that the defect transition in the $\mathrm{ZnO}$ nanorods was in resonance with the bandgap excitation of the CdSe/ZnS quantum dots, the bandgap emission of the $\mathrm{ZnO}$ nanorods was enhanced by more than a factor of 30 and the bandgap emission of the $\mathrm{CdSe} / \mathrm{ZnS}$ quantum dots was greatly reduced. The researchers believe that the enhancement involves a mechanism similar to fluorescence resonant energy transfer between the $\mathrm{ZnO}$ defect transition and the band-to-band transition in the CdSe quantum dots. This approach can become a general strategy for the design of many other nanocomposites with enhanced photoluminescence at the bandgap wavelengths.

\section{ZHAOYONG SUN}

\section{Resonant Scheme for Interferometric Lithography Improves Spatial Resolution Beyond Diffraction Limit}

M. Kiffner and J. Evers from the MaxPlanck-Institut für Kernphysik, in Heidelberg, Germany, and M. Suhail Zubairy from Texas A \& M University, USA, recently proposed a novel approach for generating subwavelength structures in interferometric optical lithography based on the preparation of the system in a position-dependent trapping state via phase-shifted standing wave patterns.

In the February 22 issue of Physical Review Letters (DOI: 10.113/PhysRevLett. 100.073602), the researchers proposed preparation of atoms in a positiondependent state by coherent population trapping. In the simplest case, two stable ground states were resonantly coupled to an excited state by laser fields with Rabi frequencies $R$ and $S$. Thus, the system was optically pumped into a coherent superposition of the two ground states, decoupled from the applied light fields. The population of each ground state depended only on the ratio of the Rabi frequencies. The researchers explained that this scheme works, in principle, for arbitrarily low laser intensities in contrast to schemes based upon multiphoton absorption, which require high field intensities. The spatial modulation was obtained by applying resonant laser fields that shift the phases of the standing waves with respect to each other such that their ratio becomes position dependent. The researchers also demonstrated that this analysis can be generalized to level schemes with an $N \times$ three-level structure. The only requirement is that all Rabi frequencies $R_{n}$ or all $S_{n}(1 \leq n \leq N)$ address different atomic transitions. A product of $N$ sinusoidal waves with wave number $k_{0}$ can lead to the population in a designated level with spatial oscillations with frequency wave number $N k_{0}$ only, cancelling all other harmonics by a suitable choice of the relative phase shifts of the standing waves.

The researchers proposed two ways for the implementation of this scheme: the use of suitable level structures in atomic gases and directly exposing a photoresist on a surface using suitable coherence times between spin states similar to that in doped solids. "Either way," the researchers said, "the applicability of our scheme already at low-field intensities considerably facilitates the realization, in particular in extended systems."

JOAN J. CARVAJAL

\section{Ants Inspire Algorithm for the Design of Conducting Polymer Alloys}

Recently, researchers at the Instituto de Física "Gleb Wataghin," Universidade Estadual de Campinas, Sao Paulo, Brazil, adapted an ant algorithm to design conducting polymer alloys. Ants establish optimal paths by depositing pheromone as they travel. The insects tend to follow trails having high pheromone concentrations, a positive-feedback mechanism termed autocatalytic. In this research, a trail is created when a virtual ant walks from one point to another on the potential surface, depositing virtual pheromone in an amount proportional to the solution at the end point. Evaporation removes pheromone from trails that are not traveled by other ants. In a given cycle, 20 ants are distributed randomly on the potential surface and are allowed to take a given number of steps. In this approach, the best trails are marked with more pheromone, because they are more traveled in the same time. The simulation ends when no improvement on the best solution is obtained in a given cycle.

As reported in the March issue of Chemical Physics Letters (p. 290), B.V.C. Martins, D.S. Galvão and co-workers considered as a test case a well-studied system of conducting polymers: polyaniline, which can have three different monomeric units. Conductivity is determined by the relative concentrations of the three forms in the polymer chain. The researchers solved the problem as a proof of concept by systematic search: determining the optimal relative concentrations of the three polyaniline forms in a polymer chain with a total of 100 monomers. Polymer bandgaps were calculated using a Hückel Hamiltonian (tight-binding model). The electron delocalization was estimated by the inverse participation number, calculat- 